\title{
In vitro degradation behavior of chitosan based hybrid microparticles
}

\author{
Ambalangodage C. Jayasuriya, Kristalyn J. Mauch
}

Department of Orthopaedics, College of Medicine, University of Toledo Health Science Campus, Toledo, USA.

Email: a.jayasuriya@utoledo.edu

Received 9 February 2011; revised 9 March 2011; accepted 21 March 2011.

\begin{abstract}
The degradation properties of the MPs is important to the long-term benefits of the use of the chitosan (CS) based hybrid MPs in bone tissue-engineering, because the degradation kinetics could affect a multitude of processes within the cell, such as cell growth, tissue regeneration, and host response. The aim of this study was to investigate the degradation of solid, hybrid CS microparticles (MPs), CS-10\% calcium phosphate $\left(\mathrm{CaHPO}_{4}, \mathrm{w} / \mathrm{w}\right)$, and $\mathrm{CS}-10 \%$ calcium carbonate $\left(\mathrm{CaCO}_{3}, \mathrm{w} / \mathrm{w}\right)$ MPs in phosphate buffered solution (PBS) over a 30-week period. The hybrid MPs were synthesized by emulsification technique, cross-linked with $64 \%$ sodium tripolyphosphate (TPP), purified and air dried overnight. Each sample had 30 mg of MPs was placed in a glass vial with $9 \mathrm{ml}$ of PBS added and then the vial was closed to prevent evaporation. Every week $4 \mathrm{ml}$ of the incubated solution was removed for sample measurement and all samples were replaced with an equivalent amount of fresh medium. The samples were maintained at $37^{\circ} \mathrm{C}$ under continuous shaking. The hybrid MPs were measured for $\mathbf{p H}$ and calcium release, every week in triplicate. At $0,5,10,15,20,25$, and 30 weeks, surface and bulk morphology were analyzed with a scanning electron microscope (SEM). The degradation data suggested that the hybrid MPs were stable at least up to 25 week and maintain the physiologically relevant $\mathrm{pH}$. Therefore, we can use these hybrid MPs to apply in the bone tissue engineering applications since they do not degrade within a short period.
\end{abstract}

Keywords: Degradation; In Vitro; Microparticles; $\mathrm{pH}$; Chitosan; Phosphate Buffered Saline

\section{INTRODUCTION}

Scaffolds are typically used in bone tissue engineering, acting as a delivery system for the cells, genetic material, and growth factors to the site of interest or defect [1].
Bone tissue engineering involves combining boneforming cells with a suitable scaffold. The scaffold is needed to function as a material for support, and a substrate for cell attachment and bone deposition $[2,3]$. The ultimate aim of bone tissue engineering is to help create a healing response in the bony area designated so that newly formed tissue would integrate with the surrounding skeleton to be useful and resilient. Ideally, the engineered scaffold will slowly degrade in a controllable manner. Controllable in the sense that degradation would match extracellular matrix formation, transfer structural and functional roles progressively to the newly formed bone while maintaining mechanical strength until tissue regeneration is almost completed, and finally be reabsorbed and metabolized by the body. The degradability of the biomaterial plays an important role in the long-term function of the engineered scaffold because it can affect many cellular process, including cell growth, tissue regeneration, and host response $[1,4,5]$. To find a suitable candidate that encompasses these aims, a variety of biomaterials have been researched for bone tissue engineering purposes, including biomaterials that can be synthetic or natural $[3,5]$.

As natural components of living structures, naturally derived polymers are of particular interest as a scaffold material for bone tissue engineering applications due to the biological and chemical similarities that they have to natural tissues. The supposition being that natural materials would have better acceptance by the human system because of its natural origins and having a closer relation to the body's original components. Chitin and its derivative chitosan (CS) are such natural biomaterials that can meet a variety of needs in the biomedical fields because their biological, physical, and chemical properties can be controlled and engineered under even mild processing conditions [4,6]. Mild processing conditions can avoid growth factor inactivation, allowing growth factors and other biomolecules to be incorporated into various composite forms for different applications [5]. Another favorable property of the derivative CS is that it can be 
molded into different forms and shapes; for example, powders, pastes, films, fibers, sponges, scaffolds, microparticles (MPs), and more have been made for various uses $[7,8]$. All these factors therefore lend credence to why chitin and CS are being increasingly studied for diverse applications as biomaterials in biomedical and pharmaceutical research, ranging from wound dressings to drug delivery carriers, as well as being used for cell encapsulation, or cartilage and bone tissue engineering $[6,9]$.

CS is a linear copolymer that is formed by the random distribution of D-glucosamine and N-acetyl-D-glucosamine residues that are linked by $\beta(1-4)$ glycosidic bonds. CS is not native to animal sources, however it is easily obtained from crustacean's exoskeletons, such as shrimp and crab, by alkaline deacetylation of chitin $[6,8,10,11]$. Removing acetyl groups from the molecular chain of chitin leaves an amino group on the chain specifically at the C-2 carbon of the glucopyranose ring in the deacetylation process of chitin to CS. However, neither chitin nor CS respectively exist $100 \%$ acetylated or $100 \%$ deacetylated, but both exist as a copolymer. Therefore, the difference between chitin and CS is the acetyl content of the copolymer. The degree of acetylation refers to the number N-acetyl-D-glucosamine units that are present and when greater than $50 \%$, the copolymer is termed chitin. When the D-glucosamine (amino groups) are predominant $(>50 \%)$ the copolymer is termed CS. The number of amino groups present refers to the degree of deacetylation [6]. Chitin's use is limited compared to CS because chitin is chemically inert and is insoluble in water and acid. CS is insoluble in neutral and basic $\mathrm{pH}$ environments $(\mathrm{pH}>7)$, and soluble in acidic environments $(\mathrm{pH}<6)$ when the amino groups become protonated to facilitate solubility $[7,12,13]$.

Besides CS being an easily obtained derivative of a natural copolymer that is moldable with reactive functionalities, the investigation into CS being used as a scaffold material in bone tissue engineering is largely due to a number of beneficial biological properties including being nontoxic, biocompatible, and biodegradable $[3,10,11,14]$. CS has been shown to have intrinsic antimicrobial actions on bacteria and fungi, to be haemostatic, antitumoral, and anticholestermic. Having an affinity to proteins, promoting cell adhesion and migration, and enhancing wound healing list a few more favorably reported properties of CS [4,11]. CS also has been shown to promote growth and mineral rich matrix deposition by osteoblasts in culture. Implants that are CS based have generally shown a minimal foreign body reaction with little or no fibrous encapsulation, which is a helpful property that could be useful to the osteointegration important in bone tissue engineering that can lend significant rigidity to bone-implant systems $[4,12$, $13,15]$.

In this study, the aim was to study the degradation behavior of hybrid MPs at physiological conditions. Since the inorganic portion of bone is composed mainly of calcium and phosphate [3], hybrid MPs were also made containing $\mathrm{CaHPO}_{4}$ or $\mathrm{CaCO}_{3}$ individually to compare and see if hybrid MPs could offer any specific advantages. Regarding this, we fabricated three different types of hybrid MPs including CS, CS- $10 \%$ calcium phosphate $\left(\mathrm{CaHPO}_{4}\right)$, and $\mathrm{CS}-10 \%$ calcium carbonate $\left(\mathrm{CaCO}_{3}\right)$ and placed in the glass vials containing phosphate buffered saline (PBS) medium with $\mathrm{pH}=7.4$. These vials containing MPs were incubated at $37^{\circ} \mathrm{C}$ with dynamic environment $(50 \mathrm{rpm})$, and refreshed medium frequently during the study. Our degradation behavior of different types of hybrid MPs was done by measuring $\mathrm{pH}$ and calcium release, and analyzing both surface and bulk morphology using scanning electron microscopy (SEM) over an extended time period of 30 weeks.

\section{MATERIALS AND METHODS}

\subsection{Materials}

CS from crab shells, practical grade and $>85 \%$ deacetylated, was purchased from Sigma-Aldrich. Other chemicals bought from Sigma-Aldrich (St. Louis, MO, USA) include: cottonseed oil, $\mathrm{CaHPO}_{4}, \mathrm{CaCO}_{3}$, sodium tripolyphosphate (TPP), Span 85, hexane, and acetic acid. Acetone was supplied by Fisher Science (Hanover, IL, USA). GIBCO PBS 7.4 (1X) liquid from Invitrogen (Grand Island, NY, USA) was used throughout the experiment. QuanticChrom calcium Assay kit was used from BioAssay Systems (Hayward, CA, USA). All solvents and chemicals used, unless specified were of analytical grade.

\subsection{Hybrid MP Preparation}

CS MPs $(1.5 \%$, w/v) were prepared by emulsification technique using our scale-up method [16,17]. CS solution was prepared by measuring $750 \mathrm{mg}$ of CS that was diluted by adding $50 \mathrm{~mL}$ of $1 \%(\mathrm{v} / \mathrm{v})$ acetic acid at room temperature. Using a stir plate, the mixture was allowed to reach homogeneity and then it was filtered through a nylon mesh 50 micron cloth to remove any remaining insoluble particulates. The CS solution $(25 \mathrm{ml})$ was quickly mixed with an equal amount $(25 \mathrm{ml})$ of acetone. This CS-acetone mixture $(36 \mathrm{ml})$ was emulsified by adding the mixture drop wise using $20 \mathrm{ml}$ syringes (Becton Dickinson [B-D], Franklin Lakes, NJ) and 20 gauge 1'1/2" needles (B-D) into mechanically stirred cottonseed oil $(600 \mathrm{ml})$ that included surfactant Span 85 $(4 \mathrm{ml})$ at $35^{\circ} \mathrm{C}-40^{\circ} \mathrm{C}$ and $850 \mathrm{rpm}$ (Corning Laboratory Stirring/Hotplate model, USA). The system continued 
stirring for $14 \mathrm{~h}$ to allow evaporation of the non-oil solvent. Then sodium tripolyphosphate (64\%, TPP) was added to allow cross-linking of the MPs for $4 \mathrm{~h}$ (Figure 1). CS MPs were washed with an equal amount of hexane $(600 \mathrm{ml})$, isolated by vacuum filtration $(11 \mathrm{~cm} \mathrm{me-}$ dium porosity filter paper, Fisherbrand, Pittsburgh, PA, USA) with a Buchner funnel, then air dried overnight. We also fabricated MPs containing $\mathrm{CaHPO}_{4}$ or $\mathrm{CaCO}_{3}$ $(10 \%, \mathrm{w} / \mathrm{w}$ solution of polymer) by adding them into the CS solution and using above described method. All prepared MPs were hydrated and neutralized in $6200 \mathrm{mg} / \mathrm{L}$ $\mathrm{NaHCO}_{3}$ for $10 \mathrm{~min}$. The solution was filtered off and the MPs were then rinsed profusely with distilled water and allowed to air-dry overnight.

\subsection{Experiment Design of in Vitro MP Degradation}

The fabricated and neutralized hybrid MPs (30 mg) were added to $11 \mathrm{ml}$ glass vial shells (Fisherbrand). MPs samples were incubated with $9 \mathrm{ml}$ of Phosphate Buffered Saline (PBS) at $37^{\circ} \mathrm{C}$ (Fisher Scientific Isotemp Incubator) under continuous shaking (Barnstead Multi-Purpose Rotator) at $50 \mathrm{rpm}$ for predetermined time points up to 30 weeks. The vials were closed with a plastic plug designed for the vials, to prevent evaporation of the PBS over the long time period of the experiment. In order to mimic in vivo physiological environment, every week 4 $\mathrm{ml}$ of incubated PBS solution was removed from each sample and replaced with an equal volume of fresh PBS medium.

\subsection{Determination of $\mathbf{p H}$}

CS and hybrid MPs were studied in triplicate samples for $\mathrm{pH}$ determination. Each week the $4 \mathrm{~mL}$ of PBS incubated solution withdrawn from the MPs was removed from their vial and placed in clean vial shells to measure the $\mathrm{pH}$ and help keep the sample from contamination. The $\mathrm{pH}$ was determined by placing a clean and calibrated $\mathrm{pH}$ meter (Mettler Toledo SevenEasy) probe into the solution then recording the stabilized value.

\subsection{Determination of Calcium Ion $\left(\mathrm{Ca}^{2+}\right)$ Release}

From the removed PBS medium used in $\mathrm{pH}$ determination, two $0.5 \mathrm{ml}$ aliquots of the incubated medium were stored at $4^{\circ} \mathrm{C}$ for assay analysis. The stored aliquots and assay kits were removed from the freezer and allowed to equilibrate to room temperature before use. The calcium assay requires preparation of a working reagent by combining equal volumes of the Reagent $\mathrm{A}$ and Reagent $\mathrm{B}$ provided. Standards, blanks, and samples were run in triplicate on clear bottom 96-well plates by adding $5 \mu \mathrm{l}$ of sample and then $200 \mu \mathrm{l}$ of working reagent to each well with tapping lightly to mix. Samples were allowed
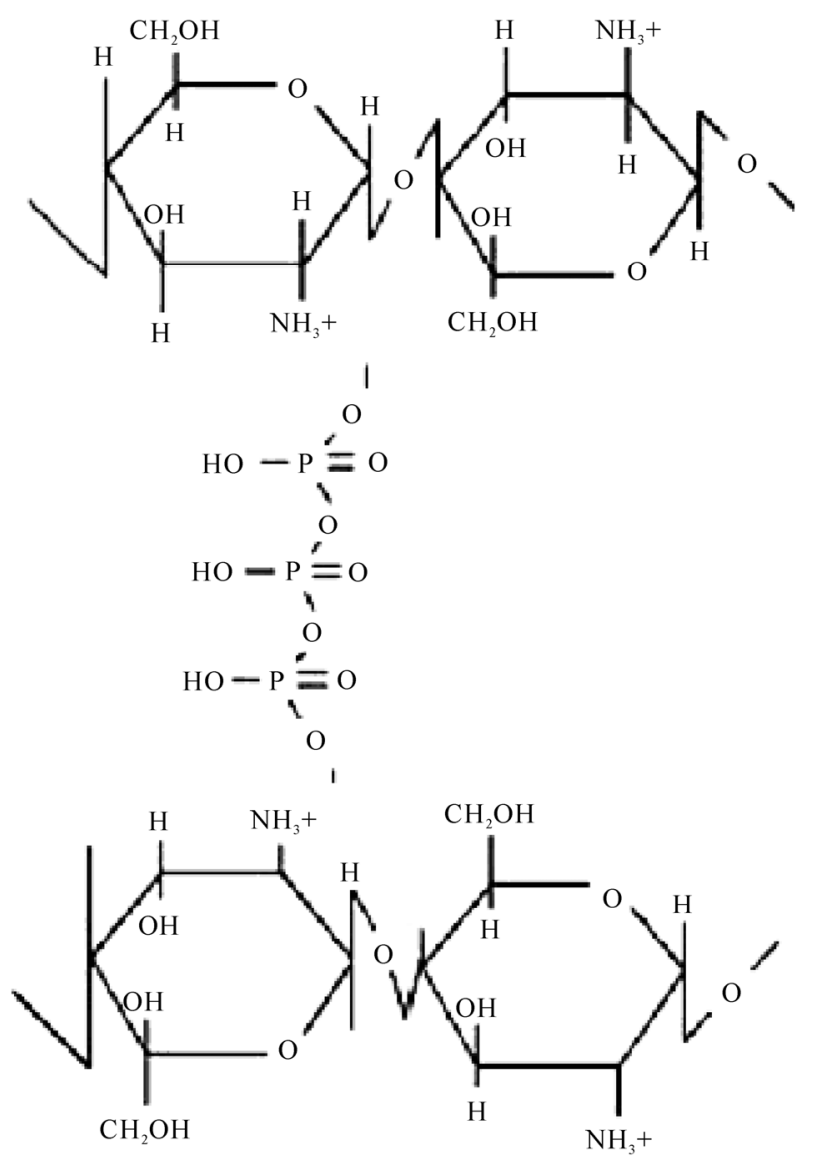

Figure 1. Ionic cross-linking interaction of amide groups in chitosan with phosphate groups in tripolyphosphate.

to incubate $3 \mathrm{~min}$ at room temperature and the optical density (OD) was read at $612 \mathrm{~nm}$ on a spectrometer (Spectra Max Plus 384, Molecular Devices; Sunnyvale, CA). Calcium concentrations of the samples were calculated with the following expression using the average OD of all triplicates of the samples:

Calcium Concentration $=\frac{O D_{\text {SAMPLE }}-O D_{\text {BLANK }}}{\text { Slope }}(\mathrm{mg} / \mathrm{dL})$

\subsection{Morphology of MPs-SEM}

At the predetermined time points of $0,5,10,15,20,25$, and 30 weeks, samples were removed for characterization by SEM. Size and morphological characteristics, including shape and surface roughness of the fabricated hybrid MPs were studied with a Hitachi S-4800 High Resolution SEM at an accelerating voltage of $10 \mathrm{kV}$. Samples for analysis with the SEM were prepared as follows: small amounts of dried MPs were affixed to a stage on double sided tape then the dry samples were gold sputtered prior to analysis with the SEM. Microphotographs were taken at different magnifications for each sample for analysis. 


\subsection{Statistical Analysis}

Statistical analysis was performed on $\mathrm{pH}$ and calcium release with a two-way ANOVA without replication through the Analysis ToolPak for data analysis in Excel. The differences were considered significant at the level of $p<0.05$.

\section{RESULTS}

\subsection{Study of $\mathbf{p H}$}

We recorded the averaged $\mathrm{pH}$ changes $(n=3)$ for the different types of MPs when they immersed in the PBS medium over the 30 weeks (Figure 2). In the figure it is clear that the MPs are causing changes in the $\mathrm{pH}$ of the PBS medium. Each MP type has a specific $\mathrm{pH}$ range that varies over time, but stays within the range that is compatible for living tissue [18]. CS MPs kept the highest $\mathrm{pH}$ range among all MP types, while CS- $10 \% \mathrm{CaHPO}_{4}$ MPs had the lowest $\mathrm{pH}$ range $(p<0.05)$ for most of the study. While CS MPs maintained the $\mathrm{pH}$ range in 7.4 7.2, $\mathrm{CS}-10 \% \mathrm{CaCO}_{3} \mathrm{MPs}$ maintained the $\mathrm{pH}$ in $7.2-7.0$. $\mathrm{CS}-10 \% \mathrm{CaHPO}_{4}$ MPs showed the lowest $\mathrm{pH}$ range, 6.8 7.0 among the three MP groups.

\subsection{Calcium Ion $\left(\mathrm{Ca}^{2+}\right)$ Release}

We fabricated MPs that integrated $\mathrm{CaHPO}_{4}$ or $\mathrm{CaCO}_{3}$ into their own respective structures as they formed. The calcium ion release in $\mathrm{mg} / \mathrm{dl}$ is reported in Figure 3 over the 25 weeks for each type of hybrid MPs $(n=3)$. There was no calcium release during the 4 weeks period from CS MPs. CS- $10 \% \mathrm{CaCO}_{3}$ MPs have the highest variation of calcium release during the initial 6 weeks compared to all the groups $(p<0.05)$. Calcium release from CS- $10 \% \mathrm{CaCO}_{3}$ MPs was decreased at 18 week and then again started to increase $(p<0.05)$. Other two types of MPs was also followed the same pattern after 18 week. Calcium release in the surface of the CS- $10 \% \mathrm{CaCO}_{3}$ MPs is seems to be faster than compared to that of CS- $10 \% \mathrm{CaHPO}_{4} \mathrm{MPs}$.

\subsection{Morphology Change-SEM}

The size, shape, and surface roughness of MPs can influence cell attachment, proliferation, and differentiation [2]. SEM microphotographs were used to determine these characteristics of hybrid MPs. Almost prepared MPs appeared to be spherical and distinctively separate at Week 0, which means just after placing the MPs in the vials containing PBS for $10-15$ min but not yet started to incubate them (Figures 4-6). All MPs types were measured had their smallest diameter approximately 20 $\mu \mathrm{m}$. The most CS MPs were in the size range between $20-75 \mu \mathrm{m}$. However, there were a few MPs were over the upper limit of the size range. The $\mathrm{CS}-10 \% \mathrm{CaCO}_{3}$ and $\mathrm{CS}-10 \% \mathrm{CaHPO}_{4} \mathrm{MPs}$ were slightly larger compare to the CS MPs and the most MPs were in the size of 20 $90 \mu \mathrm{m}$, and $20-100 \mu \mathrm{m}$, respectively. Similar to CS MPs there were a few large $10 \% \mathrm{CaCO}_{3}$ and CS- $10 \%$ $\mathrm{CaHPO}_{4} \mathrm{MPs}$ in the out of above size range.

In Figure 4, microphotographs show the changes in surface morphology of CS MPs with low (50X) and high magnification (700X). Week 0 had smooth, spherical morphology with slight ridges elevations. At Week 5 we noticed a change to the outer surface morphology of most CS MPs. Larger ridges and wrinkling were observed. The wrinkles seem to become more predominant as the time points passed. At Week 25, we were interested that the microphotographs revealed that the some MPs were peeling but the shape was not affected. At week 30, small pieces were broken from the some CS MPs. However, we did not observe the complete bulk degradation of MPs suggesting that bulk degradation initiate at this time period.

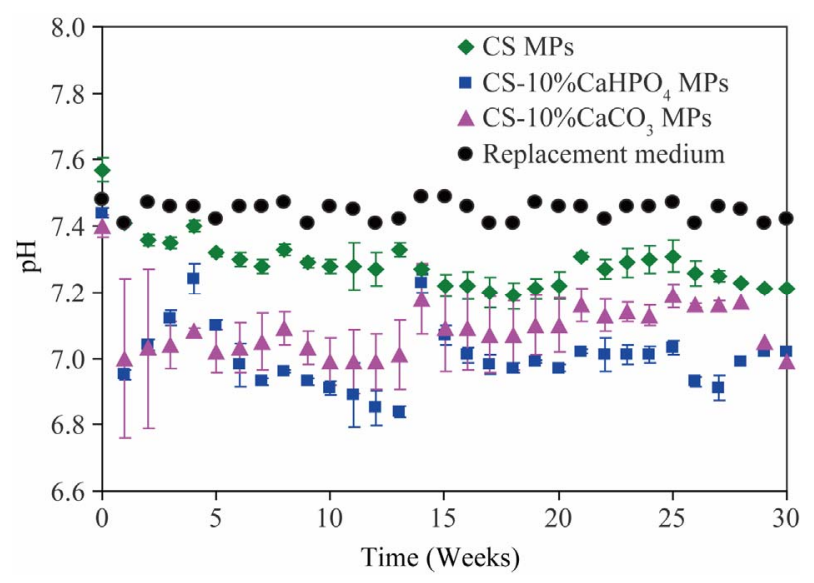

Figure 2. The $\mathrm{pH}$ data for hybrid MPs as a function of time up to 30 week. The line with solid spheres shows the $\mathrm{pH}$ of the replacement PBS medium used.

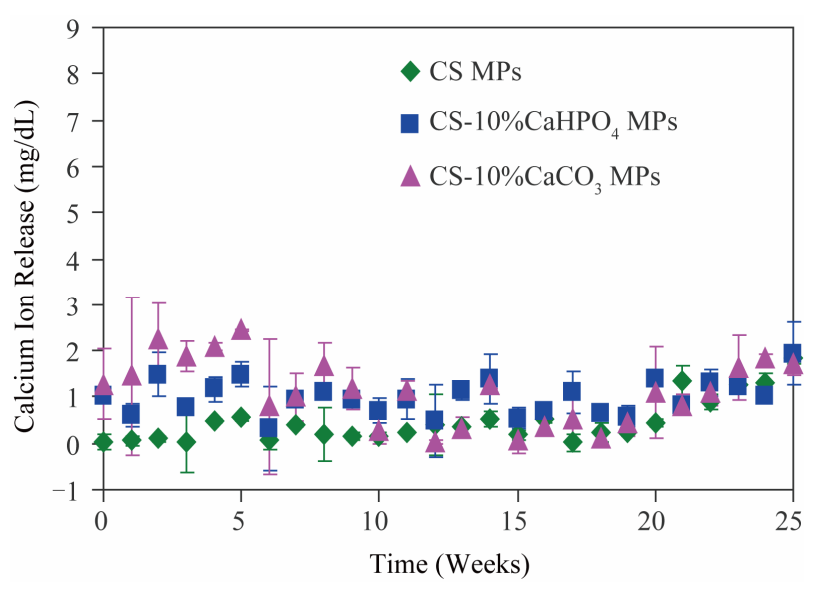

Figure 3. Calcium release data for each type of MPs over the 25-weeks. 

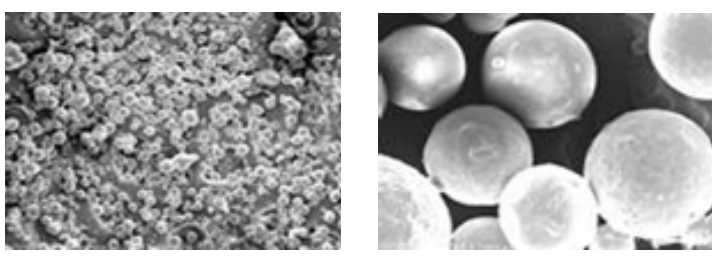

(a)
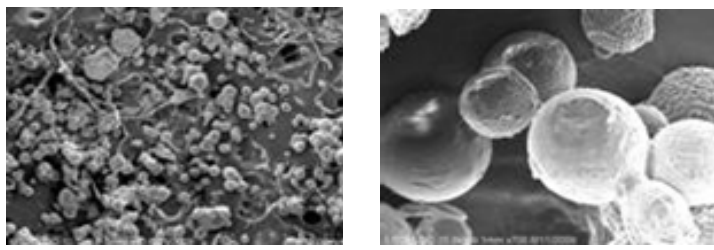

(b)
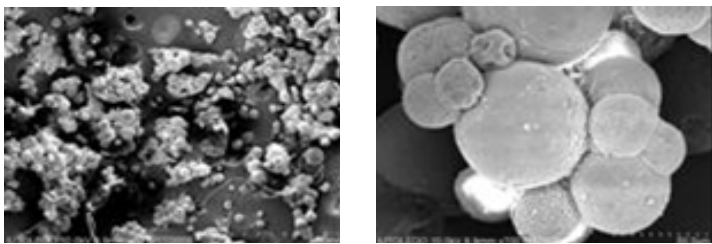

(c)
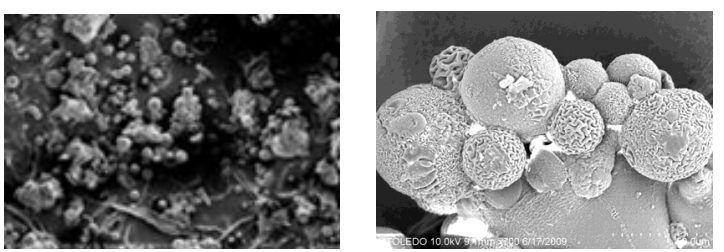

(d)
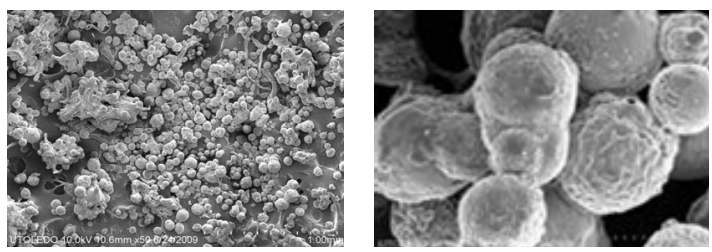

(e)
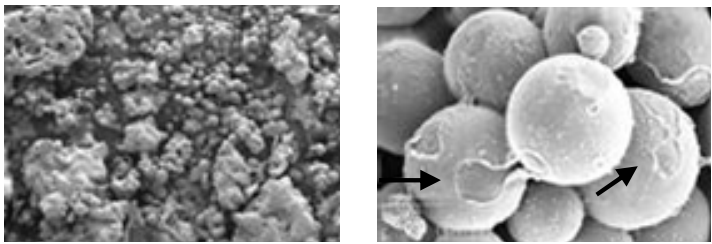

(f)
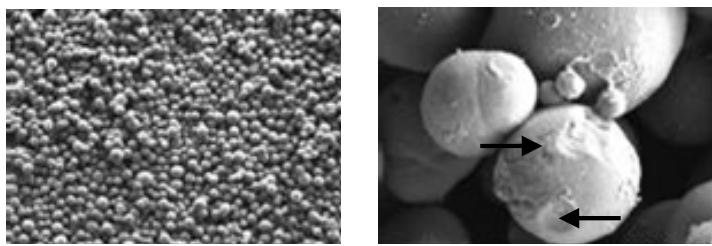

(g)

Figure 4. SEM microphotographs of CS MPs from weeks (a) 0 ; (b) 5; (c) 10; (d) 15; (e) 20; (f) 25; and (g) 30. 1 and 2 denote low (50X) and high (700X) magnification, respectively. Arrows indicate the degradation started areas.
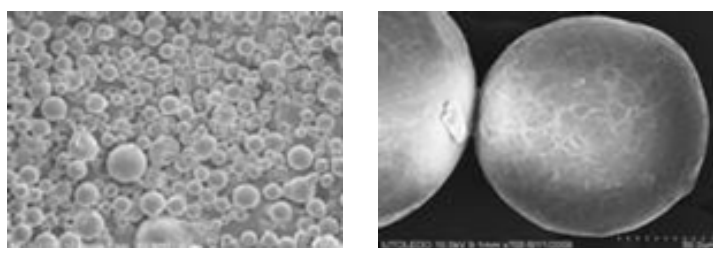

(a)
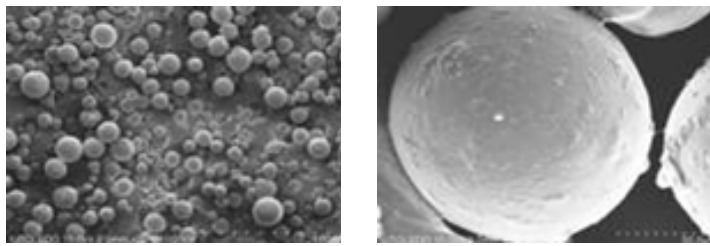

(b)
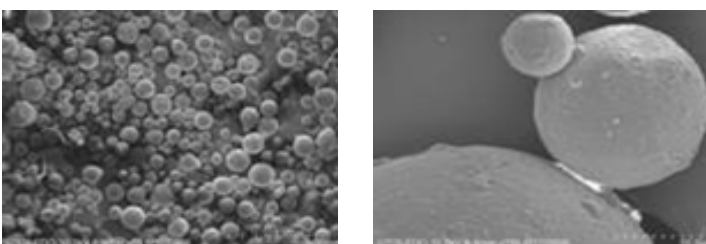

(c)
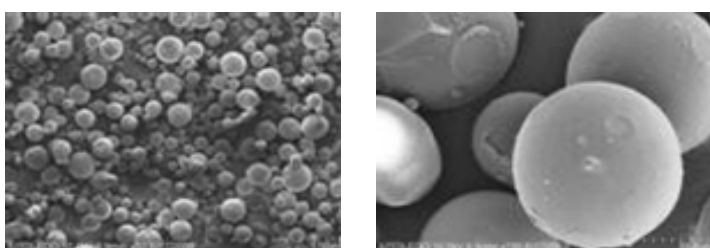

(d)
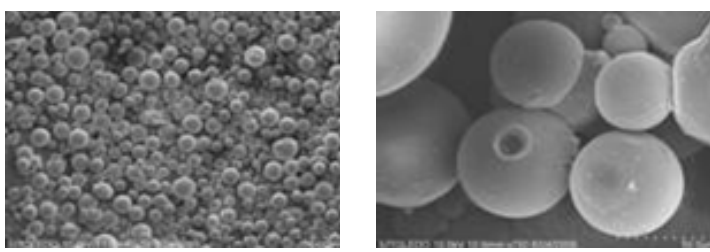

(e)
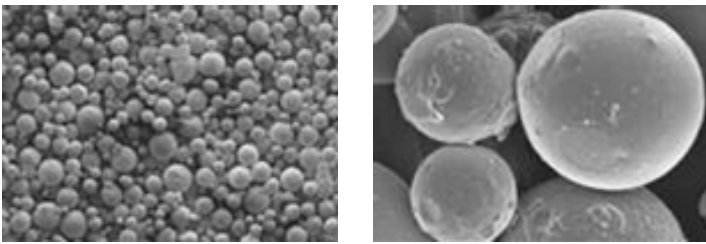

(f)
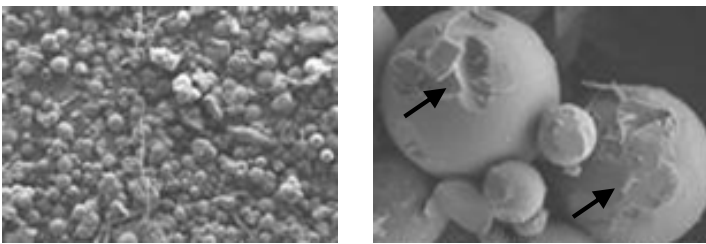

(g)

Figure 5. SEM microphotographs of CS-10\% $\mathrm{CaHPO}_{4}$ MPs from weeks (a) 0; (b) 5; (c) 10 ; (d) 15 ; (e) 20; f) 25; and (g) 30.1 and 2 denote low (50X) and high (700X) magnification, respectively. Arrows indicate the degradation started areas. 

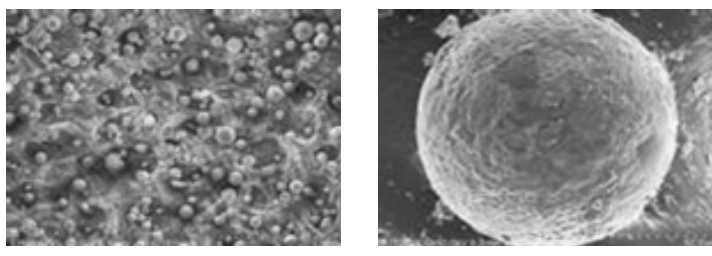

(a)
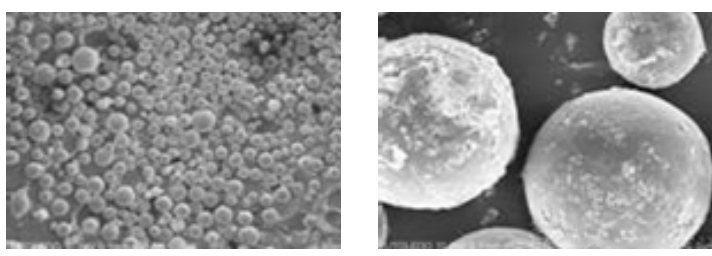

(b)
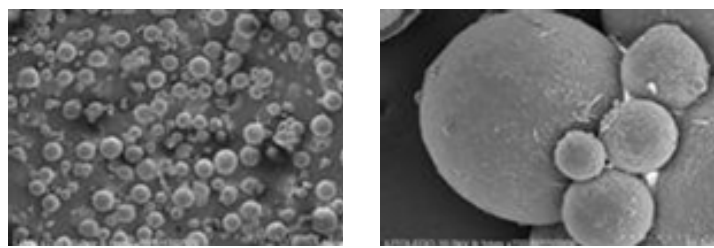

(c)
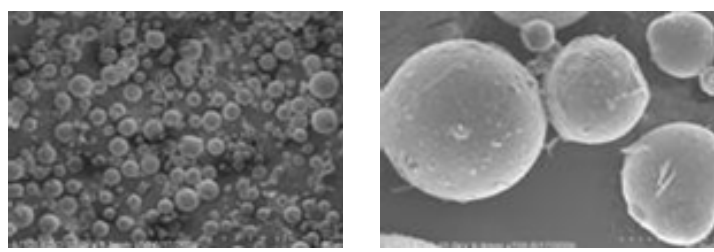

(d)
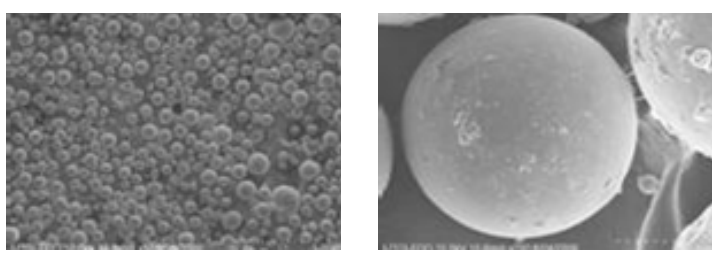

(e)
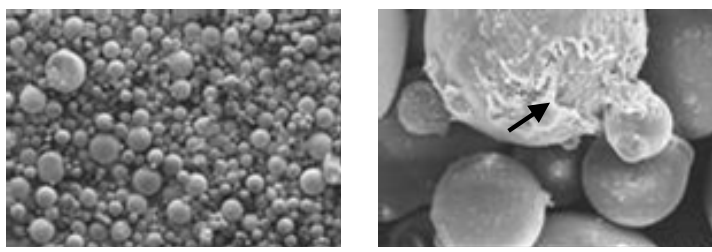

(f)
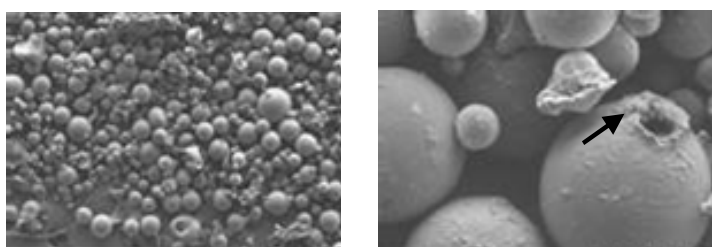

(g)

Figure 6. SEM microphotographs of $\mathrm{CS}-10 \% \mathrm{CaCO}_{3}$ MPs from weeks (a) 0; (b) 5; (c) 10; (d) 15; (e) 20; f) 25; and (g) 30. 1 and 2 denote low (50X) and high (700X) magnification, respectively. Arrows indicate the degradation started areas.
Microphotographs of CS-10\% $\mathrm{CaHPO}_{4}$ MPs (Figure 5) revealed spherical particles that tended to stay separate, but have an increasingly rougher surface with the passage of time. However, the surface is considerably smoother than CS MPs. Small holes can also be observed in the CS- $10 \% \mathrm{CaHPO}_{4}$ MPs especially after Week 20. At week 25, the particle size and shape were not affected but at week 30, the pieces of the most particles were observed suggesting the initiation of bulk degradation.

Figure 6 shows the $\mathrm{CS}-10 \% \mathrm{CaCO}_{3}$ MPs microphotographs (50X and 700X) that reveal a distinctive flaky Week 0 appearance. Beginning Week 5 the noticeable flaky appearance was mostly gone, exhibiting a smoother surface that was more comparable to CS MPs from week 0 . The flakiness appearance went away and the surface did get mildly rougher over time. The initial flakiness may be attributed to the leaching of $\mathrm{CaCO}_{3}$, TPP, or both. At 25 and 30 weeks, breaking of parts and appearing of holes can be visible in some $\mathrm{CS}-10 \% \mathrm{CaCO}_{3}$ MPs.

\section{DISCUSSION}

We fabricated the ionically cross-linked hybrid MPs (Figure 1) in this study using our optimized scale up method as described previously [16]. We also characterized these hybrid MPs in terms of chemical composition and ionically cross-linked structure, physical structure and morphology using different analytical techniques [17]. The hybrid MPs are spherical in shape with smooth outer surface without porous structure. The most MPs fabricated were in the range of $20-100 \mu \mathrm{m}$.

Instead of static conditions of the degradation, we performed degradation studies similar to in vivo conditions, for example, kept the vials containing MPs in PBS at $\mathrm{pH} 7.4$ in a shaking table $(50 \mathrm{rpm})$ inside the incubator at $37^{\circ} \mathrm{C}$. The incubated medium in the vials was also refreshed frequently. Before using these hybrid MPs to study drug release profiles or in vivo studies, it is necessary to know their biodegradation behavior in the physiological environment.

The $\mathrm{pH}$ data of hybrid MPs during 30 week period was in the range of $6.8-7.4$. The CS MPs has shown the highest $\mathrm{pH}$ values $(7.2$ - 7.4) during the 30 week period suggesting that $\mathrm{CS}-10 \% \mathrm{CaHPO}_{4} \mathrm{MPs}$ involves slightly lower values $(6.8-7.0)$ of $\mathrm{pH}$ compared to other two types of MPs. These lower $\mathrm{pH}$ values of $10 \% \mathrm{CaHPO}_{4}$ MPs may be related to the release of $\mathrm{H}+$ ions from $\mathrm{CaHPO}_{4}$ in $10 \% \mathrm{CaHPO}_{4}$ MPs. Hydrogen ion concentrations outside of the tenfold $\mathrm{pH}$ range of 7.8 to 6.8 are not compatible with living tissue if maintained [18]. The $\mathrm{pH}$ of these hybrid MPs over 30 week period fulfilled the above requirement. However, we anticipate that we can improve the higher $\mathrm{pH}$ values if we use less acetic acid when we fabricate the MPs. In addition we must use the 
lyophilizer to get rid of any remaining residual acetyl group in the MPs.

We examined the calcium release for all types of MPs during the 25 week period (Figure 3). The calcium release from MPs was less than $2 \mathrm{mg} / \mathrm{dl}$ at a time during the 25 week period. This released calcium amount seems to be less than the calcium content in the human serum which is $8.5-10.5 \mathrm{mg} / \mathrm{dl}$ [19]. Our MPs contain only $10 \%(\mathrm{w} / \mathrm{w})$ of $\mathrm{CaHPO}_{4}$ or $\mathrm{CaCO}_{3}$ relative to CS amount. We can anticipate that higher release of calcium at a time if we incorporate the higher amount of calcium containing compounds into the hybrid MPs. The initial calcium release seems to be attributing to the diffusion and later release after 18 week may be due to the more degradation of the MPs. The CS MPs may not possess calcium since we did not incorporate any materials containing calcium. However, our data has shown the calcium release over time from CS MPs. We would like to note that Chestnutt et al. [20] also reported the interesting release of calcium in pure CS scaffolds. A fact that was attributed to the use of CS from crab shells containing residual mineral content.

SEM data (Figures 4-6) has clearly shown that these MPs did not change its shape or size until 25 week. At or after 25 week all types of MPs seem to show the initiation of bulk degradation. Generally, the degradation of CS is related to the molecular weight and deacetylation. These MPs were strong not only having the higher deacetylation $(>85 \%)$ but these MPs were ionically cross-linked with TPP. This interaction leads to the fabrication of physically strong particles according to our optimization and scale-up procedures [16].

It has been demonstrated that CS can be degraded enzymatically by chitinases, chitosanases, and lysozyme. Past research has shown CS to be degraded mainly by lysozyme in human serum [21,22]. Lysozyme appears to target the acetylated units on CS, leaving CS oligosaccharides products of different lengths that can be incorporated into glycosaminoglycans and glycoproteins pathways or excreted $[13,23]$. Many investigations have published that the factor in controlling the rate of degradation has been shown to be inversely associated with CS's degree of deacetylation. Highly acetylated CS degrades rapidly, and highly deacetylated $(>80 \%)$ CS has low degradation rates and may remain several months in vivo $[5,6,10,12,13,22]$.

There is an increased demand towards surgical interventions being minimally invasive. Preferable scaffold constructs in bone tissue engineering for such surgeries would be moldable and/or injectable to the defect site. Some studies have shown the possibility of such osteogenic constructs with pre-cultured ceramic MPs. However, low biodegradability and brittleness can restrict the use of ceramics $[2,4]$. A goal of our studies is to help characterize and design CS based hybrid MPs that could become an injectable biomaterial suitable for a scaffold-like role in bone tissue engineering. We believe it to be important to understand the process that is happening to the MPs without enzyme for background results that can be used comparatively with what happens with enzymatic degradation in future studies.

\section{CONCLUSIONS}

In this study using physiological conditions we have shown that morphological changes occur on the surface followed by initiation of bulk degradation of $85 \%$ deacetylated CS based hybrid MPs without enzyme hydrolysis over 30 week period. The size and shape of the hybrid MPs were not affected until week 30 . The broken pieces were observed at Week 30 from the all types of hybrid MPs. Our results show that each MP type maintain the physiologically relevant $\mathrm{pH}$ range during the 30 week period. We conclude that investigation of using CS based hybrid MPs for a bone tissue engineering purpose is encouraged by our biodegradation results, and the feasibility of using these MPs for a long-term scaffold-like role is possible.

\section{ACKNOWLEDGEMENTS}

We would like to thank National Science Foundation (NSF) grant number 0652024 and National Institute of Health (NIH) grant number DE019508 for providing financial support to accomplish this work.

\section{REFERENCES}

[1] Kakar, S. and Einhorn, T.A. (2005) Chapter 11: Tissue engineering of bone. In: T. Einhorn \& J. O. Hollinger, Eds., Bone Tissue Engineering, CRC Press, Boca Raton, 277-302.

[2] Kruyt, M.C., Persson, C., Johansson, G., Dhert, W.J. and De Bruijn, J.D. (2006) Towards injectable cell-based tissue-engineered bone: The effect of different calcium phosphate mps and pre-culturing. Tissue Engineering, 12 , 309-317. doi:10.1089/ten.2006.12.309

[3] Zhang, Y. and Zhang, M. (2001) Synthesis and characterization of macroporous CS/calcium phosphate composite scaffolds for tissue engineering. Journal of Biomedical Materials Research, 55, 304-312. doi:10.1002/1097-4636(20010605)55:3<304::AID-JBM1 018>3.0.CO;2-J

[4] Kim, I.Y., Seo, S.J., Moon, H.S., Yoo, M.K., Park, I.Y. and Kim, B.C. et al. (2008) Chitosan and its derivatives for tissue engineering applications. Biotechnology Advances, 26, 1-21.

doi:10.1002/1097-4636(20010605)55:3<304::AID-JBM1 $018>3.0 . \mathrm{CO} ; 2-\mathrm{J}$

[5] Jiang, T., Kumbar, S.G., Nair, L.S. and Laurencin, C.T. (2008) Biologically active CS systems for tissue engineering and regenerative medicine. Current Topics in Medicinal Chemistry, 8, 354-364. 
doi:10.2174/156802608783790974

[6] Khor, E. (2001). Chitin: Fulfilling a biomaterials promise. Elsevier, New York.

[7] Shi, C., Zhu, Y., Ran, X., Wang, M., Su, Y. and Cheng, T. (2006) Therapeutic potential of CS and its derivatives in regenerative medicine. Journal of Surgical Research, 133, 185-192. doi:10.1016/j.jss.2005.12.013

[8] Kato, Y., Onishi, H. and Machida, Y. (2003) Application of chitin and CS derivatives in the pharmaceutical field. Current Pharmaceutical Biotechnology, 4, 303-309. doi:10.2174/1389201033489748

[9] Abdel-Fattah, W.I., Jiang, T., El-Bassyouni, G.E.T. and Laurencin, C.T. (2007) Synthesis, characterization of CSs and fabrication of sintered CS microsphere matrices for bone tissue engineering. Acta Biomaterialia, 3, 503-514. doi:10.1016/j.actbio.2006.12.004

[10] Freier, T., Koh, H.S., Kazazian, K. and Shoichet, M.S. (2005) Controlling cell adhesion and degradation of CS films by N-acetylation. Biomaterials, 26, 5872-5878. doi:10.1016/j.biomaterials.2005.02.033

[11] Couto, D.S., Hong, Z. and Mano, J.F. (2009) Development of bioactive and biodegradable CS-based injectable systems containing bioactive glass nanoparticles. Acta Biomaterialia, 5, 115-123. doi:10.1016/j.biomaterials.2005.02.033

[12] Di Martino, A., Sittinger, M. and Risbud, M.V. (2005) Chitosan: A versatile biopolymer for orthopaedic tissue-engineering. Biomaterials, 26, 5983-5990. doi:10.1016/j.biomaterials.2005.03.016

[13] Francis Suh, J.K. and Matthhew, H.W. (2000) Application of CS-based polysaccharide biomaterials in cartilage tissue engineering: A review. Biomaterials, 21, 25892598. doi:10.1016/S0142-9612(00)00126-5

[14] Anal, A.K., Stevens, W.F. and Remunan-Lopez, C. (2006) Ionotropic cross-linked CS microspheres for controlled release of ampicillin. International Journal of Pharmaceutics, 312, 166-173. doi:10.1016/j.ijpharm.2006.01.043

[15] Costantino, P.D., Hiltzik, D., Govindaraj, S. and Moche, J. (2002) Bone healing and bone substitutes. Facial Plastic Surgery, 18, 13-26. doi:10.1055/s-2002-19823
[16] Jayasuriya, A.C. and Bhat, A. (2009) Optimization of scaled-up chitosan microparticles for bone regeneration, Biomedical Materials, 4, 55006. doi:10.1088/1748-6041/4/5/055006

[17] Jayasuriya, A.C. and Bhat, A. (2010) Fabrication and characterization of novel hybrid organic/inorganic microparticles to apply in bone regeneration. Journal of Biomedical Materials Research A, 93A, 1280-1288.

[18] Widmaier, E.P., Raff, H. and Strang, K.T. (2006). Chapter 2: Chemical composition of the body. In: Vander, Ed., Human Physiology: The Mechanisms of Body Function, McGraw-Hill, Boston, pp. 21-46.

[19] Seltzer, M.H., Rosato, F.E. and Fletcher, M.J. (1970) Serum and tissue in human breast carcinoma. Cancer Research, 30, 615-616.

[20] Chesnutt, B.M., Viano, A.M., Yuan, Y., Yang, Y., Guda, T., Appleford, M.R. et al. (2009) Design and characterization of a novel CS/nanocrystalline calcium phosphate composite scaffold for bone regeneration. Journal of Biomedical Materials Research Part A, 88A, 491-502. doi:10.1002/jbm.a.31878

[21] Vårum, K.M., Myhr, M.M., Hjerde, R.J., Smidsrød, O. (1997) In vitro degradation rates of partially N-acetylated CSs in human serum. Carbohydrate Research, 299, 99-101. doi:10.1016/S0008-6215(96)00332-1

[22] Ren, D., Yi, H., Wang, W. and Ma, X. (2005) The enzymatic degradation and swelling properties of chitosan matrices with different degrees of $\mathrm{N}$-acetylation. Carbohydrate Research, 340, 2403-2410. doi:10.1016/S0008-6215(96)00332-1

[23] Tığlı, R.S., Karakeçili, A. and Gümüşderelioğlu, M. (2007) In vitro characterization of CS scaffolds: Influence of composition and deacetylation degree. Journal of Materials Science: Materials in Medicine, 18, 1665-1674. doi:10.1007/s10856-007-3066-X

[24] Young, M.A., Ravishanker, G., Beveridge, D.L. and Berman, H.M. (1995) Analysis of local helix bending in crystal structures of DNA oligonucleotides and DNArotein complexes. Biophysical Journal, 68, 2454-2468. doi:10.1016/S0006-3495(95)80427-3 